\title{
Estilos vinculares y conducta alimentaria en niños
}

\section{Binding styles and children nurturing behavior}

\author{
Alfonso Cofré Lizamaa, Javier Moreno Obrequea, Felipe Salgado Péreza, Alex Castillo Delgadoa y Enrique Riquelme \\ Mellab, \\ aUniversidad Santo Tomás, Temuco \\ bUniversidad Católica, Temuco \\ (Rec.: agosto de 2017 - Acept.: noviembre de 2017)
}

Resumen

Esta investigación tuvo como objetivo explorar la relación entre estilos vinculares y conducta alimentaria en niños y niñas de 6 a 9 años. Se exploran diferencias en estilos vinculares, índice de masa corporal y conducta alimentaria, además de diferencias por género. La muestra fue de carácter no probabilístico y estuvo constituida por 100 niños pertenecientes a la ciudad de Temuco. Se utilizan los instrumentos TFEQ-P19 para medir conducta alimentaria y el test del Dibujo de la Familia para explorar el apego. Por último, el peso y talla se obtuvo a través de las mediciones efectuadas a los participantes para el posterior cálculo de IMC. Los resultados indican que existe un $15 \%$ de participantes con sobrepeso y $42 \%$ en estado de obesidad. Los resultados indican que existe una relación significativa entre estilo vincular y estado nutricional, observando una asociación entre hombres con estilo vincular evitante y obesidad. El abordaje de la obesidad también puede ser considerado desde las relaciones tempranas, como un eje articulador significativo entre la dimensión emocional e ingesta alimentaria.

Palabras Claves: Apego, Conducta alimentaria, Niños, Obesidad

\begin{abstract}
The purpose of this research was to explore the relationship between binding styles and nurturing behavior in boys and girls in a range of age from 6 to 9 years old. Differences in binding styles, body mass index, nurturing behavior and gender differences are explored. The sample was off non-probabilistic nature and constituted by 100 children belonging from Temuco city. The instruments TFEQ-P19 were used to measure nurturing behavior and The Family Drawing test to explore attachment. Finally, weight and size was obtained through the measures carried out to the participants for the following BMI calculation. The results indicate that $15 \%$ of the participants were overweight and $42 \%$ were obese. The results point out that there is a meaningful relationship between avoiding binding style and obesity. Dealing with obesity can be considered since early relationships as a significant basic premise between emotional dimension and food intake.
\end{abstract}

Key Concepts: Attachment, Eating behavior, Children, Obesity

* Correspondencia: Dr. Alfonso Cofré Lizama. Univesidad Santo Tomás, Chile. Email. acofrel@gmail.com 


\section{Introducción}

Estilos vinculares y conducta alimentaria en niños.

La obesidad es un problema de salud pública a nivel mundial, afectando a países de alto, mediano y bajos ingresos (Organización Mundial de la Salud [OMS], 2015). Esta patología es conceptualizada como una enfermedad multifactorial (Pataky, Robbioni- Harsh \& Golay, 2010). La OMS (2015) estima que en el año 2010 ya existían 42 millones de niños con sobrepeso en todo el mundo, de los que aproximadamente 35 millones pertenecen a países en vías de desarrollo. La Organización de las Naciones Unidas para la Alimentación y la Agricultura (FAO, 2015) sitúa a Chile con una de las tasas más altas de obesidad infantil entre los países de la región, llegando al 10\% de la población, siendo específicamente el segundo país con más niños obesos menores de 5 años. Por otra parte, Atalah (2012) demuestra una situación similar, donde sitúa en un $23,1 \%$ a la población obesa entre los 6 y 7 años.

Sobre las enfermedades asociadas a la obesidad, se estima que entre el $25 \%$ y el $30 \%$ de los pacientes obesos, presentan algún trastorno psicológico como depresión, ansiedad, trastorno por atracón, conducta de evasión y agresión, marginación social y baja autoestima (Vásquez, 2004).

Los programas de salud buscan prevenir la obesidad y/o estimular la pérdida de peso, centrándose principalmente en la promoción de la actividad física y el consumo de alimentos saludables (Organización Mundial de la Salud, 2015). Existen diversas teorías asociadas a la adquisición de la obesidad. Entre estas, destaca la teoría de los genes expuesta por Manco y Dallapiccola (2012), quienes plantean que la heredabilidad a la susceptibilidad de adquirir la enfermedad es relativamente alta. Desde una perspectiva social, Christakis y Fowler (2007) destacan la importancia de los vínculos sociales como variable explicativa del aumento de peso. Cruz, Tuñón, Villaseñor, Álvarez y Nigh (2013) afirman que -desde la perspectiva biomédica- el sobrepeso y la obesidad es una enfermedad que conlleva factores de riesgo para la aparición de otras patologías. Peña y Bacalao (2000) afirman que existe una asociación entre pobreza y obesidad, destacando el factor socioeconómico en relación con la enfermedad.

Guillén (2014) enfatiza en el rol de la familia, mencionando que para evitar conflictos se utiliza el alimento como un mecanismo desviador de tensiones y ansiedades. Por otro lado Van der Merve (2007), se refiere a la obesidad como un conflicto psíquico, que no puede ser resuelto por los mecanismos de defensa.

Desde una perspectiva constructivista, se resalta la importancia que existe en las relaciones de reciprocidad temprana y los desórdenes alimenticios psicógenos, señalando que la modulación de ritmos corporales básicos como el hambre y la motilidad, proporcionan la única posibilidad para una autopercepción fiable dentro de una experiencia inmediata en continua vacilación, lo cual reduciría la probabilidad de sentimientos de vacío (Guidano, 1994). Según Zagmutt (2010), en las relaciones afectivas ocurre una especie de sintonía emocional que hace que la regulación emocional de cada individuo dependa en alto grado del vínculo con las figuras significativas. Arciero (2004) plantea que, para mantener una cierta proximidad, los niños regulan la relación con la figura de apego tratando de correspon- der en cada momento a las expectativas de este, caracterizando así un patrón más pasivo a través de la ingesta alimentaria. La Teoría del Apego (Bowlby, 1982) señala que los seres humanos buscan la cercanía a otras personas significativas a través de un sistema psicobiológico y adaptable en momentos en que está presente la sensación de vulnerabilidad. Las interacciones que se producen con figuras de apego estables y la sensación de seguridad, implica promover un apoyo real y simbólico para el aprendizaje de estrategias de autorregulación (Mikulincer \& Shaver, 2012). Según Cassidy (1994), estas interacciones entregan a los niños herramientas para comprender lo que es socialmente aceptable, expresar sus emociones y entender los sentimientos de los demás. Específicamente en un marco familiar, donde sus dinámicas de comunicación sean ambiguas y contradictorias, el propósito primario es proveer la imagen de un matrimonio feliz, evitando expresiones externas de emociones definidas u opiniones que pudieran indicar la existencia de un problema o insatisfacción recíproca (Guidano, 1987). Por lo tanto, debido a que procesos psicológicos como regulación emocional, estilos afectivos, autoestima, y autoimagen, se originan en la formación de estilos de apego, es que se hace necesario buscar patrones de conducta restrictiva o sobrealimentación influyentes en los procesos mencionados (Montalvini, Lucero \& López, 2014).

Henríquez, Basso y Álves (2015), establecen asociaciones significativas entre apego inseguro y obesidad, concluyendo que los factores emocionales también están implicados en la etiología y el mantenimiento del sobrepeso. Por otra parte, Faber (2015) señala que el apego de los padres inseguros, se asocia con una mayor ingesta de alimentos obesogénicos. Por último, una investigación realizada por Anderson y Whitaker (2011), obtuvieron como resultado que en niños con apego inseguro se observa un $23,1 \%$ de prevalencia de obesidad, mientras que el $16,6 \%$ de niños obesos mostraban apego seguro. Estos resultados enfatizan la importancia de lograr una sana relación cuidador-niño fomentando la relevancia en la confianza de los padres, la disponibilidad y sensibilidad. Sin embargo, las cifras apuntan a una problemática de magnitud, y no se ha realizado una investigación de similares características en la novena región, considerando además, que presenta patrones culturales propios de la localización geográfica, que diferenciaría de otras investigaciones.

Con respecto a lo anterior, este estudio pretende como objetivo general "Explorar la relación entre estilos vinculares y conducta alimentaria en niños y niñas de 6 a 9 años", y como objetivos específicos los siguientes: a) explorar diferencias en estilos vinculares, IMC y conducta alimentaria en niños y niñas con bajo peso, normopeso y sobrepeso y, b) explorar las diferencias de género en estilos vinculares y su relación en la conducta alimentaria en niños y niñas de 6 a 9 años con bajo peso, normopeso y sobrepeso.

\section{Antecedentes generales}

La obesidad en términos conceptuales, se caracteriza por el alto contenido de grasa corporal, que se distribuye según volumen y topográficamente en el cuerpo (Moreno, 2012). Esta enfermedad se considera de origen multifactorial, existiendo un aumento de la cantidad de grasa relativa a la talla corporal de la persona (Morales, Santos, González, Ho \& Hodgson, 2012). 
El parámetro más utilizado para medir el sobrepeso y la obesidad es el índice de masa corporal (IMC), cuya determinación es sencilla y permite identificar el sobrepeso y la obesidad en los adultos, tanto a nivel individual como poblacional. El IMC se define como el peso en kilogramos dividido por el cuadrado de la talla en metros (kg/m2)"(OMS, 2015). El producto de esta operación matemática igual o superior a 25 , determina un sobrepeso, y un IMC igual o superior a 30 determina una condición de obesidad (OMS, 2015).

\section{Prevalencia}

En el año 2010 ya existían 42 millones de niños con sobrepeso en todo el mundo, de los que 35 millones pertenecen a países en vías de desarrollo (OMS, 2015). En Europa la prevalencia de sobrepeso en niños de 7 y 11 años oscila del 10 al 35\%, y en adolescentes la prevalencia oscila del 8 al 23\%. En EE.UU. la prevalencia de sobrepeso y obesidad también se ha duplicado en las últimas dos décadas, siendo actualmente del 15\% (Azcona, Romero, Bastero \& Martínez, 2005).

En países latinoamericanos la prevalencia oscila entre el 22 y 26\% en Brasil, 21\% en México, 10\% en Ecuador, 22\% en Perú, $22 \%$ en Paraguay y un $27 \%$ en Argentina (Braguinsky, 2009). La FAO (2015) sitúa a Chile con una de las tasas más altas en el contexto de obesidad infantil, específicamente el segundo país con más niños obesos menores de 5 años. Así, la población infantil chilena, tiene una prevalencia de $20 \%$ en los últimos años en niños de primero básico pertenecientes a escuelas públicas; porcentaje que aumenta si se considera la adolescencia (Castillo-Durán, Le Royo \& Osorio, 2012).

A nivel regional, un estudio que el Ministerio de Salud (2013), revela que la región de la Araucanía se encuentra entre las cinco regiones con mayores índices de obesidad infantil en el país, con un 12,1\% de prevalencia en menores de 6 años. Además, la comuna de Temuco se ubica como la segunda en el país con mayor cantidad de niños obesos, siendo sólo superada por la comuna de Puente Alto, en Santiago (Ministerio de Salud de Chile [MINSAL], 2013).

\section{Problemas asociados}

Se estima que entre el $25 \%$ y el $30 \%$ de los pacientes obesos, muestra algún trastorno psicológico, como depresión, ansiedad, trastorno por atracón, conducta de evasión y agresión (Vásquez, 2004). En niños, el sobrepeso puede generar baja autoestima, por lo cual su imagen corporal es negativa y el rendimiento académico e interacción social se ven afectados por los síntomas depresivos y ansiosos ante su condición física (Karnik \& Kanekar, 2012).

\section{Teorías que explican la obesidad}

Manco y Dallapiccola (2012), enfatizan la influencia de la herencia, que varía según aspectos como la dieta de la madre durante la gestación o el desarrollo prenatal del niño. Desde una perspectiva social, Christakis y Fowler (2007) afirmaron modelos estadísticos longitudinales con la finalidad de determinar si la obesidad pudiese tener un componente ambiental que cumpla el rol de factor de riesgo ante padecer esta enfermedad, arrojando como resultado que los vínculos sociales serían una variable explicativa del aumento de peso de las personas. Por otra parte, Cruz et al (2013), establecen que -desde el paradigma biomédico- se considera al sobrepeso y la obesidad como una enfermedad que conlleva factores de riesgo para la aparición de otras patologías. Por su parte Peña y Bacallao (2001), refieren que existe una asociación entre pobreza y obesidad, exponiendo el factor socioeconómico como una posible explicación a este problema de la salud.

Un enfoque nutricional propone que los cambios en los patrones alimentarios como el alto consumo de azúcares, grasas, así como el pobre consumo de vegetales, frutas, y fibras son los principales causantes de esta epidemia (Peña \& Bacallao, 2001).

\section{Conducta alimentaria}

La conducta alimentaria es el conjunto de acciones que establecen la relación del ser humano con los alimentos. Se acepta generalmente que los comportamientos frente a la alimentación se adquieren a través de la experiencia directa con la comida, por la imitación de modelos, la disponibilidad de alimentos, el estatus social, los simbolismos afectivos y las tradiciones culturales. Además de las influencias sociales, se ha señalado que las influencias genéticas y de ambiente familiar compartido, tienen un impacto relevante sobre la conducta alimentaria y la obesidad infantil (Domínguez-Vásquez, Olivares \& Santos, 2009).

\section{Apego}

Existen diversas variables que pueden contribuir al mantenimiento de la obesidad. Es el caso de algunas personas que no logran adecuar en su desarrollo una manera adaptable de controlar su alimentación, en donde los padres inicialmente juegan un rol importante ya sea en la niñez o cuando hay sobreprotección (Berch, 2006); por otra parte, hay autores que plantean que en el mundo occidental, específicamente desde las primeras etapas del desarrollo, se recurre mayormente a la comida para atenuar el malestar emocional. En tanto Castillo y Romo (2006), explican que existen elementos en la comida que producen placer. Esto no se reduce solo a ingerir un determinado tipo de alimento, sino que están también el goce de compartir la comida, el placer de cocinar para los que se quiere (mostrándoles afecto de esta manera), y el placer obtenido en la adquisición de la comida a través de actividades colectivas. Según Gonzales y Martínez (2011), comer también sería una fuente de placer, lo cual condiciona la selección de alimentos según sus características organolépticas; de esta manera, las experiencias placenteras o aversivas relacionadas con la ingesta de ciertos alimentos pueden llevar a conductas reforzadas que se repiten a lo largo de los años. Finalmente, la alimentación además es un acto que se basa en normas socialmente aceptadas y que cumple un rol de relación entre las personas que conforman una misma cultura (Gonzales \& Martínez, 2011).

De acuerdo a esto, la importancia que cobran los factores socio-culturales en relación a las prácticas alimentarias pueden ser determinantes, así mismo, estos modos de alimentarse, preferencias y rechazos hacia determinados alimentos, están fuertemente condicionados por el contexto familiar, siendo la etapa infantil donde se incorpora la mayoría de los hábitos y prácticas alimentarias de la comunidad (Domínguez-Vásquez, Olivares \& Santos, 2009). En esta lógica, es importante explorar cómo los cuidadores regulan emocionalmente a sus 
hijos y cómo las experiencias tempranas de vinculación pueden influir en su desarrollo psicológico y emocional, teniendo en cuenta que el desarrollo es proactivo e influido por condiciones intersubjetivas e ineludibles basadas en las relaciones madre-hijo, lazos sociales, cohesión de grupo, entre otras (Guidano, 1994). Por lo tanto, el sentirse emocionalmente involucrado en la percepción de una semejanza o reciprocidad con otros seres significativos, parece ser la condición esencial que subyace en todo desarrollo del conocimiento ontológico durante el desarrollo vital de un ser humano (Guidano, 1994).

Mediante esta perspectiva, es necesario hablar sobre el modelo propuesto por Bowlby (1982), el cual señala que los seres humanos buscan la cercanía a otros significativos (como figuras de apego), a través de un sistema psicobiológico y adaptable, en diversos momentos que presentan cualquier sensación de vulnerabilidad. De esta manera plantea la existencia de cuatro sistemas de conductas relacionados entre sí: el sistema de conductas de apego, el sistema de exploración, el sistema de miedo a los extraños y el sistema afiliativo. En tanto, el sistema de apego refiere a todas las conductas que pretenden un mantenimiento de la proximidad y contacto con las figuras de apego, comportamientos que se activan cuando hay un aumento de distancia con la figura de apego o cuando se perciben señales de amenazas. Contrario a esto surge el sistema de exploración, el cual aparece cuando las conductas de apego se ven disminuidas. Paralelamente, el sistema de miedo muestra una relación con los anteriores ya que su aparición supone la disminución de las conductas exploratorias y activación de las conductas de apego. Por último, el sistema afiliativo refiere al interés que los seres humanos demuestran por mantener proximidad e interactuar con otros sujetos, incluso con quienes no han establecido vínculos afectivos.

Para Bowlby, el modelo representacional viene a ser una representación mental de sí mismo y de las relaciones con otros seres significativos. Este modelo denominado "Modelo operante de apego" se va a construir principalmente por las relaciones con las figuras de apego, lo cual le va a servir al sujeto para percibir e interpretar las acciones e intenciones de los demás y por último, dirigir de esta manera su conducta como una predisposición. Estas conductas permiten establecer relaciones significativas entre los cuidadores y los niños, y entre los adultos, surgen a partir de representaciones mentales internalizadas, definidas por Bowlby (1982) como pautas o estilos de apego. En el caso de los adultos, estas reciben el nombre de modelos internos o mapas representacionales de las relaciones (Pinedo Palacios, y Santelices Álvarez, 2006). En términos prácticos, lo que transmite este modelo es que incluye componentes activos y cognitivos, teniendo así una noción de quiénes son las figuras de apego, dónde se pueden encontrar y qué se espera de ellas. También contiene información acerca de uno mismo, por tanto constituye una base propia de identidad y de la autoestima.

A través de diversos estudios, particularmente como el de Schaffer y Emerson (1964), se fue demostrando que el tipo de vínculo que los niños establecían con sus cuidadores, iba a depender principalmente de la sensibilidad de estos adultos con respecto a las demandas o peticiones del niño. Dentro de esta misma línea Ainsworth y Bell (1970), realizaron una investigación en la que se expuso a la madre y el niño ante una persona extraña, evidenciando que el niño utiliza a la madre o cuidador como una base segura para la exploración, y que la sensación de cualquier amenaza activaba las conductas de apego y hacía desaparecer las conductas exploratorias que el niño podía tener. En esta misma lógica Ainsworth (1969), encontró tres patrones principales de apego: niños de apego seguro que lloraban poco y se mostraban contentos cuando exploraban en presencia de la madre, niños de apego inseguro, que lloraban frecuentemente aún estando en brazos de sus madres y por último, niños que parecían no mostrar apego ni otro tipo de conductas diferenciales hacia su madre (Oliva, 1995).

\section{Obesidad y apego.}

En este contexto, los autores Anderson y Whitaker (2011) concluyeron que la prevalencia de obesidad fue del $23,1 \%$ en niños con apego inseguro y el $16,6 \%$ en niños con apego seguro. Para los niños con apego inseguro, las probabilidades de obesidad fueron de 1,30 veces por sobre los niños con apego seguro después de controlar la calidad de la interacción madrehijo durante el juego, la crianza de las prácticas relacionadas con la obesidad, el índice de masa corporal de la madre, y las características socio demográficas.

Otro estudio realizado (Broberg, Hjalmers, \& Nevonen, 2001), consideró desórdenes alimenticios y el apego en pacientes de 18 a 24 años de edad, arrojando como resultado que efectivamente existe una relación entre los trastornos de la alimentación, el apego inseguro y problemas interpersonales, indicando además, que la gravedad de los trastornos alimentarios está directamente relacionada con la seguridad del primer vínculo.

En tanto Kiesewetter et al. (2012), trabajaron con 44 pacientes obesos que participaron en un programa de reducción de peso multimodal de un año. El estilo de apego fue analizado por el inventario de Apego Adulto Prototipo Valoración (AAPR) y su relación con un programa de reducción por un año. Los resultados muestran que el estilo de apego seguro estuvo en el $68 \%$ de los participantes, y el inseguro (preocupado y desprendido), en el $32 \%$. Curiosamente se encontró una reducción de peso significativamente mayor en forma segura (SAI), en comparación con los individuos apego inseguro (UAl; $p<0,05$ ). Esta estimación también correlacionó positivamente con calidad de la alianza paciente-terapeuta ( $p$ =0,004). Es decir, los vínculos de apego temprano tienen una estrecha relación con la capacidad de autorregulación en la alimentación, mostrando además la importancia del vínculo con el terapeuta.

Frankel, Hughes, O'Connor, Power, Fisher, y Hazen (2012), determinan a partir de su investigación sobre apego entre padres e hijos, que la autorregulación emocional que los niños aprenden de padres inseguros puede afectar los patrones de ingesta de alimentos y ser un factor de riesgo para la obesidad infantil, ya que una mala regulación genera respuestas de estrés, lo que se relaciona con la obesidad.

Al respecto, podemos decir que existen estudios que han abordado la dinámica entre apego y obesidad en distintos países y con diferentes edades, sin embargo, en la novena región no se ha realizado una investigación de estas características, y teniendo en cuenta que las cifras de obesidad en la Araucanía son alarmantes, se hace necesaria una mayor profundización en cuanto a las causas de la obesidad, abarcando no solo as- 
pectos alimenticios y de actividad física. De igual manera, aun pudiendo realizar un trabajo de la misma temática y similar metodología a otros estudios previos, el nuestro sería de gran aporte, ya que se descubrirá cómo se relaciona esta dinámica según patrones culturales propios de la localización geográfica.

\section{Método}

\section{Participantes}

La muestra fue constituida por 100 niños, cuyas edades oscilaron entre los 5 y 9 años (= 7; DT $=0.9$ ), quienes cursaban entre primer y tercer año de enseñanza básica en un establecimiento educacional de la ciudad de Temuco. Del total de participantes, 55 fueron hombres (55\%) y 45 fueron mujeres (45\%). La muestra fue de carácter no probabilístico. En primera instancia se solicitó la autorización a entidades responsables de la escuela, vale decir, al director, al jefe de UTP y a los profesores con jefatura de los cursos seleccionados para el estudio. Para llevar a cabo el levantamiento de información, todos los participantes que aportaron a la investigación, fueron previamente autorizados por sus padres a través de cartas de consentimiento informado y asentimiento de los niños.

\section{Instrumentos}

La conducta alimentaria se midió en base al cuestionario de alimentación de tres factores (Three factor eating questonaire TFEQ-P19) desarrollado por De Lauzon et al. (2004). Esta es una versión adaptada de la original TFEQ, de 51 preguntas para estudios epidemiológicos, aplicable a sujetos obesos y normopeso, mediante la información entregada por los progenitores. El TFEQ evalúa 3 dimensiones de la conducta alimentaria: "restricción cognitiva" (6 preguntas), "alimentación sin control" (10 preguntas) "alimentación emocional" (3 preguntas). Las respuestas se miden en una escala Likert que abarcan desde 1 (completamente cierto) a 4 (completamente falso), y los puntajes estandarizados de cada dimensión -en ambos cuestionarios-, se calcularon como la suma de las preguntas de cada dimensión dividida por el número de preguntas, debido a que no existen puntos de corte establecidos con fines diagnósticos. Así mismo, el análisis es realizado mediante una interpretación general por cada dimensión respecto a la conducta alimentaria. En esta muestra, la consistencia interna del instrumento fue la siguiente: en su dimensión "restricción cognitiva" presenta una consistencia de 0.70 , mientras que "variable emocional", presentó 0.73 y en "alimentación sin control", un 0.71. Todos estos datos referentes al alfa de Cronbach.

El segundo instrumento aplicado en la investigación, que medió la variable apego, fue el test del Dibujo de la familia, utilizando el Sistema de Kaplan y Main para su interpretación. Este método contiene un conjunto de descripciones de funciones del dibujo y cómo estas características se modelan para los cuatro principales grupos de clasificación del test, que son: seguro (B), inseguro- evitativo (A), inseguro- ambivalente (C), o inseguro- desorganizado (D). En el contexto escolar (específicamente en el aula), se pidió a los niños que efectúen el dibujo dándole la consigna correspondiente y las instrucciones de la tarea, entregándoles una hoja en blanco para realizar dicho dibujo y lápices de colores. Se requirió de 3 jueces para interpretar el dibujo, quienes -en una primera instancia- analizaron el gráfico individualmente, para luego compartir sus análisis de manera de llegar a un consenso en la interpretación final, considerando la presencia o ausencia de las descripciones de funciones según el sistema de Kaplan y Main.

Sobre el índice de confiabilidad, la codificación se llevó a cabo por dos jueces independientes y posteriormente, por un tercer juez para así resolver los casos de desacuerdo. En un primer nivel, se realizó un análisis gráfico y formal, y se evaluaron por separado los aspectos de contenido de los dibujos (de acuerdo al sistema de Kaplan y Main). En un segundo nivel, los dibujos fueron evaluados mediante escalas de ocho de cinco puntos de calificación global (Procaccia, Veronese \& Castiglioni, 2014). Cabe señalar que esta metodología fue aplicada en un estudio en Milano, Italia. La consistencia interna del instrumento fue de, 973 .

\section{Procedimiento}

En el marco de un diseño descriptivo-comparativo, primero se contactó a las autoridades del colegio para solicitar el acceso a la muestra. Se presentó el proyecto, sus objetivos, sus fines y sus requerimientos para poder hacer a sus estudiantes partes de la muestra investigativa, esto en una reunión con la directora del establecimiento. Posteriormente, se tomó contacto con los docentes de Educación Física para coordinar los días y horarios en los que se tomarían mediciones de talla y peso, en conjunto con la aplicación del test de dibujo de la familia. Los padres de los niños que conformaron la muestra fueron informados de los objetivos y fines del proyecto mediante cartas de consentimiento informado, las cuales nos permitieron proceder con las respectivas actividades. La muestra fue pesada y medida durante el período de clases en grupos de cinco alumnos (con el fin de no entorpecer el desarrollo de esta), utilizándose una huincha de medición y una pesa calibrada. Con la información recopilada, se procedió a calcular el IMC de cada sujeto para así clasificar su estado nutricional (saludable - obeso - sobrepeso - obeso ND + 100). Este procedimiento contó con la aprobación del Comité de Ética de la Universidad Santo Tomás, considerándose que todo paciente firmara un consentimiento informado antes del estudio.

El test TFEQ-P19 se le hizo llegar a los padres por medio de sus hijos para que lo devolviesen contestado.

El Dibujo de la familia fue realizado por los menores en la sala de clases, para que así los niños(as) realizaran la actividad en un contexto conocido y sin muchos distractores, esto con los permisos previos de la directiva del establecimiento y del determinado docente del curso.

\section{Resultados}

Para dar respuesta al objetivo 1, el cual buscó explorar diferencias en estilos vinculares y conducta alimentaria en niños de 6 a 9 años con normopeso, bajo peso y sobrepeso, se procedió inicialmente a establecer una comparación de promedios de índice de masa corporal según estilo vincular. Los resultados señalan diferencias estadísticamente significativas entre IMC y Apego, donde el valor de Chi cuadrado con la prueba Kruskal-Wallis corresponde a 9.47 (3); p=0.02. La comparación por parejas evidencian diferencias estadísticamente significativas 
entre el apego seguro y apego evitativo: $U=<20,13 ; p=0.03$. Un segundo análisis de este primer objetivo, consistió en comparar el IMC según estado nutricional (obeso, normopeso, sobrepeso). Los resultados indican que existen diferencias estadísticamente significativas con Kruskal-Wallis, que corresponde a 7,2 (2); $p<0.01$. La comparación por parejas entrega diferencias estadísticamente significativas entre los tres estados nutricionales. Complementariamente, se realizó la comparación del estado nutricional y la conducta alimentaria. Los resultados señalan diferencias estadísticamente significativas entre cada una de las dimensiones y el estado nutricional. Los valores de las pruebas estadísticas pueden ser observados en la tabla 1.

Tabla 1. Significación por dimensión (TFEQ-P19)

\begin{tabular}{lcc}
\hline Dimensión & Valor Prueba & Significación \\
\hline Alimentación Emocional & 8,596 & 0,014 \\
Alimentación Sin Control & 7,216 & 0,027 \\
Restricción Cognitiva & 6,274 & 0,043 \\
\hline
\end{tabular}

Respondiendo este mismo objetivo específico, se procedió a comparar las dimensiones de la conducta alimentaria en función del apego, y los resultados señalaron que no existen diferencias estadísticamente significativas, entre conducta alimentaria y estilo vincular. Los valores pueden ser observados en la tabla 2.

Tabla 2. Dimensiones TFEQ-P19 en función de apego

\begin{tabular}{lcc}
\hline Dimensión & Valor Prueba & Significación \\
\hline Alimentación Emocional & 5,56 & 0,13 \\
Alimentación Sin Control & 3,34 & 0,34 \\
Restricción Cognitiva & 0,8 & 0,8 \\
\hline
\end{tabular}

Para complementar este análisis, se procedió a establecer una comparación entre estilo vincular y estado nutricional. Los resultados indican que las variables son dependientes entre sí con prueba $\chi^{2}(6)=22,69 ; p=0.001$; con $V$ de Cramer $=0.33$; $p=0.01$. Esto es considerado una correlación significativa.

Para responder al segundo objetivo específico, el cual señala "Explorar las diferencias de género en estilos vinculares y su relación en la conducta alimentaria en niños de 6 a 9 años con bajo peso, normopeso y sobrepeso", se procedió a comparar el IMC según género. Los resultados no señalan diferencias estadísticamente significativas. En efecto, los hombres obtuvieron un rango promedio de 51.29 y las mujeres de 48.46; el valor de $U$ fue de 1145,5 con $p=0.625$. Al explorar estilo vincular según género se puede establecer una asociación con la prueba $\chi^{2}(3) 14.91 ; p=0.02$.

Al explorar potenciales diferencias entre género y conducta alimentaria no se observan diferencias estadísticamente sig- nificativas. Posteriormente, se procedió a explorar la relación entre la variable género y estado nutricional, donde la prueba $\chi^{2}$ no señala una asociación estadísticamente significativa: $\chi^{2}$ $(2)=5.04 ; p=0.08$.

Finalmente, se procedió a realizar un análisis de estilo vincular según estado nutricional en función del género. Los resultados indican que se observa una asociación en hombres (prueba $\chi^{2}=15.90 ; p=0.014$ ) y no se diferencian en mujeres (prueba $\chi^{2}=7.11 ; p=0.13$ ).

Las medidas direccionales de tau de Goodman-Kruskal señalan que el $3 \%$ de error se ve reducido al predecir el valor de la variable dependiente estado, conociendo del valor de la variable independiente apego. La posibilidad de disminuir el error es de 0.09 al poner al apego como variable dependiente del estado nutricional.

\section{Discusión}

El objetivo de esta investigación pretendió medir la relación entre estilos vinculares y conducta alimentaria en niños y niñas de 6 a 9 años en un establecimiento educacional de la ciudad de Temuco, con el fin de contribuir al abordaje terapéutico de la obesidad entendiendo al apego como una variable significativa en el proceso de construcción, regulación emocional y reciprocidad de cada niño con el cuidador y su relación con los alimentos.

Durante los primeros años de la infancia (entre los seis y siete años, paralelo al aumento de la capacidad cognitiva y motriz), crece la aptitud para manipular activamente la inmediatez de la modulación emocional, a fin de mantener coherentemente la evaluación del sí mismo. Respecto a esto, fue necesario conocer los estilos vinculares predominantes en relación con el estado nutricional que presentó la muestra evaluada. De este modo, el estudio se basó en las premisas de la teoría de apego planteada por John Bowlby (1958), así como los lineamientos propuestos por el modelo postracionalista de Guidano (1983). Los resultados señalan que existe una relación significativa entre estilo vincular y estado nutricional (bajo peso, normopeso, sobrepeso, obeso), hallazgo que concuerda con lo expuesto por Arias y Gutiérrez (2014) que obtuvieron como resultado que el estilo de apego hacia la madre, padre y pares en adolescentes de 14 años, tiene una relación significativa con las alteraciones en los patrones de la conducta alimentaria. Se encontró que los sujetos con sobrepeso, obesidad y desnutrición presentan un estilo de apego evitativo. En cuanto al presente estudio se observa una asociación en hombres específicamente, de estilo vincular evitante y obesidad, no así en mujeres. La posibilidad de disminuir error se reduce en un $13 \%$ cuando estado nutricional queda como variable dependiente de apego. Estos resultados concuerdan con hallazgos similares, donde dan cuenta de asociaciones significativas entre el apego inseguro y la obesidad, tomando nota de que estos factores emocionales también están implicados en la etiología y el mantenimiento de exceso de peso (Da cruz et al., 2015).

En relación a los resultados encontrados, podemos ver que existen correlaciones estadísticamente significativas entre IMC y estilo vincular; así mismo, la variable IMC también se relaciona de manera significativa con el estado nutricional de la muestra. Igualmente, la variable estado nutricional tuvo implicancias significativas al ser relacionada con la conducta 
alimentaria, la cual fue analizada por sus respectivas dimensiones (Alimentación emocional, Alimentación sin control y Restricción cognitiva)

Similares conclusiones obtuvo Anderson, Gooze, Lemeshow \& Whitaker (2012), mediante la realización de un estudio en el cual su objeto fue determinar si la obesidad en la adolescencia está relacionada con la calidad de la relación materno-infantil temprana. Los resultados arrojaron que la mala calidad de la relación madre-hijo temprana se asoció con una mayor prevalencia de obesidad adolescente. Por otra parte, Valladares et al. (2015), también establecen diferencias significativas entre los puntajes de conducta alimentaria en niños y niñas obesos, en donde para la dimensión de "alimentación emocional" (medido con el cuestionario TFEQ-P19) los niños presentan mayores puntajes $(p=0.01)$. En este estudio también se encontraron asociaciones significativas con algunas dimensiones de la conducta alimentaria relacionadas con la sensación de saciedad.

Por su parte, el estilo vincular y estado nutricional indican que las variables son dependientes entre sí, por lo que es considerado una correlación significativa. No obstante, entre las dimensiones de la conducta alimentaria en función al estilo vincular, no se presentan diferencias estadísticamente significativas. Teniendo en cuenta lo anterior, los datos indican que en relación al estado nutricional, $15 \%$ de la muestra se encuentra con sobrepeso y un $42 \%$ en estado de obesidad. Actualmente, no existen datos sobre prevalencia de obesidad en niños del rango etario observado, por lo que la última cifra obtenida corresponde a un estudio realizado por Atalah (2012), que menciona que los índices de sobrepeso y obesidad superan el $40 \%$ en niños que ingresan a enseñanza básica. Sin embargo, las tres dimensiones del instrumento (TFEQ-P19) no entregan asociaciones significativas entre estilo vincular y conducta alimentaria. Sobre esto, se podría hipotetizar que hubo cierta distorsión al momento de contestar los cuestionarios por parte de los padres en relación a la percepción que tienen sobre la corporalidad de sus hijos, la forma de alimentarse o la relación con la comida. En la misma línea, Angarita, Martínez, Rangel, Betancourt y Prada (2014), observan en su investigación que más del $50 \%$ de los padres no perciben correctamente el peso de sus hijos, por lo que el reconocimiento de la obesidad no sería del todo objetiva.

Por otra parte, a pesar que el instrumento posee una confiabilidad interna adecuada, probablemente no es lo suficientemente sensible para lograr especificar categorías de conducta alimentaria. Bajo la mirada de lo estadísticamente descriptivo junto a la teoría del apego, se evidencia en la muestra un predominio de $61 \%$ de niños con un estilo vincular inseguro, del cual $28 \%$ sería evitativo, $28 \%$ ambivalente y $5 \%$ desorganizado. En contraste, Anderson (2011) en un estudio longitudinal de 977 niños, observó que el $24.7 \%$ de ellos presentaba una mala calidad de las relaciones materno-infantil, en comparación con el $22.0 \%$ que no tuvo un tipo de apego inseguro ni una baja sensibilidad materna. En la adolescencia, la probabilidad de obesidad era de 2.45 veces mayor en aquellos con una mala calidad de las relaciones materno-infantil en comparación con los niños que tuvieron mejor vínculo de apego. Por lo anteriormente expuesto, podríamos inferir que la proyección en términos de salud de estos niños sería más bien negativa y con ciertas implicancias en su organización personal. Dentro de esta perspectiva Hesse (1999), plantea que en las representaciones de los sujetos con estilo de apego inseguro se evidencian ciertas fallas a nivel de integración de información contradictoria y dolorosa. Una característica vincular insegura-evitativa, implicaría por un lado una minimización de los afectos y vivencias negativas, y por otro, un énfasis en la autonomía. Atendiendo estas consideraciones, otras investigaciones plantean que establecer estilos vinculares de tipo inseguro tendría ciertas incidencias en el bienestar psicológico, por ejemplo, la presencia de un patrón de apego evitativo, que se caracterizaría por una negación de necesidad de otro y una excesiva autonomía, algo que podría relacionarse con la resistencia a continuar ciertos tratamientos en algunos pacientes. Desde un polo más ansioso-ambivalente, existiría una alta preocupación por las relaciones y necesidad de otros para regular sus propias emociones (Biedermann, Martínez, Olhaberry \& Cruzat, 2009).

Los resultados obtenidos al comparar IMC según género, indican que no existen diferencias estadísticamente significativas. De igual manera al explorar diferencias entre género, conducta alimentaria y estado nutricional, tampoco señalan una asociación significativa, lo que concuerda con los resultados obtenidos por Coromoto, Pérez, Herrera, \& Hernández (2011), quienes no encontraron diferencias estadísticas de género entre hábitos alimentarios, estado nutricional y actividad física en preescolares, poniendo énfasis en la relación de hábito alimentario y estado nutricional donde tampoco se encontraron asociaciones significativas.

Finalmente, al proceder a analizar el estilo vincular según estado nutricional en función del género, los resultados indican una asociación en hombres y no en mujeres. Con esto podríamos hipotetizar que existen diferencias en cuanto a la regulación afectiva por parte de los padres o cuidadores en relación al género. En esta lógica las niñas serían tratadas de manera más adecuada según las necesidades psicológicas que respectan a cada una. Atendiendo a los supuestos anteriores, las investigaciones actuales en Chile no se ajustan específicamente a los rangos etáreos propuestos por este estudio, no obstante, podemos tener una aproximación general desde el cuarto estudio de maltrato infantil en Chile (UNICEF, 2015) en donde los hallazgos develan un contraste en relación a la violencia (psicológica, física leve y física grave) ejercida por ambos padres según género, resultando así ser las mujeres más vulneradas en comparación a los hombres.

De acuerdo a los descriptivos de este estudio, el 56\% de la muestra se encuentra en un estado nutricional de sobrepesoobesidad, y en contraste con datos entregados por el último informe mapa nutricional de JUNAEB, tales estadísticas superan el promedio de estado nutricional en niños de primero básico (51,8\% en sobrepeso). Esto quiere decir que en relación al nivel país, la muestra en cuestión está por sobre el promedio obtenido por el Ministerio de Educación (2013).

Por todo lo anterior, consideramos que los planes de intervención respecto a la obesidad infantil en nuestro país no están abordando esta problemática de manera integral, pudiendo estos niños transformarse en adolescentes obesos, tal como plantean Carrasco, Gomez y Staforelli (2009), indicando que los adolescentes obesos poseen una historia de aumento de peso de larga data, asociadas a síntomas ansiosos y depresivos. Atendiendo estas consideraciones, la perspectiva de la teoría del apego nos entrega bases sólidas para comprender 
el rol fundamental que las figuras significativas tienen a la hora de establecer vínculos estables con sus hijos y cómo estos podrían influir en su regulación emocional y a la vez, en la relación con la ingesta alimentaria. De las evidencias anteriores, futuras investigaciones deberían considerar distinciones en las variables socioeconómicas, así como patrones culturales que puedan influir en una muestra determinada.

\section{Referencias}

Ainsworth, M.D.S.(1969). Object relations, dependency, and attachment: A theoretical review of the infantmother relationship. Child Development, 40, 9691025.

Ainsworth, M. D. S., \& Bell, S. M. (1970). Attachment, exploration, and separation: Illustrated by the behavior of one-year-olds in a strange situation. Child Development, 41, 49-67.

Anderson, S., \& Whitaker, R. (2011). Attachment Security and Obesity in US Preschool-Aged Children. Arch Pediatr Adolesc Med, 165(3), 235-242.

Anderson, S., Gooze, R., Lemeshow, S., \& Whitaker, R. (2012). Quality of Early Maternal-Child Relationship and Risk of Adolescent Obesity. Pediatrics, 129, 129-132.

Angarita, A., Martínez, A., Rangel., Betancourt, M. y Prada, G. (2014), Determinantes de la subestimación de la percepción de los padres sobre el peso de sus hijos en hogares infantiles del ICBF de Floridablanca, Colombia, 2012. Revista Chilena de Nutrición, $41,372-382$

Arciero, G. (2004). Estudios y diálogos sobre la identidad personal: reflexiones sobre la experiencia humana. Islas Canarias: Ilustre Colegio Oficial de Psicólogos de las palmas.

Arias, L., Gutiérrez, M. (2014). Estilos de apego y actitudes hacia la alimentación en adolescentes (Tesis para optar al título de licenciado en psicología). Universidad Autónoma del Estado de México, México. Recuperado desde http://ri.uaemex.mx/handle/20.500.11799/41199

Atalah, E. (2012). Epidemiología de la Obesidad en Chile. Revista Medica Clínica Las Condes 117-123.

Azcona S., Romero M., Bastero, M. E., \& Martínez. (2005). Obesidad infantil. Revista Española de Obesidad, 3 (1) 26-39.

Biedermann, K., Martínez, V., Olhaberry, M., \& Cruzat, C. (2009). Aportes de la Teoría del Apego al Abordaje Clínico de los Trastornos de Alimentación. Revista Argentina de Clínica Psicológica, 18 , 13,217-226. Recuperado de: http://www.redalyc.org/articulo. oa?id=281921775003.

Bowlby, J. (1958). The nature of the child's tie to his mother. Internacional Journal of PsychoAnalysis , 39, 350-373
Bowlby, J. (1982).Attachment and Loss. New York: Basic Books.

Braguinsky, J. (2009). Prevalencia de obesidad en América Latina. Anales Del Sistema Sanitario De Navarra, 25, 109-115. Recuperado de http://recyt.fecyt.es/ index.php/ASSN/article/view/5493/4534

Broberg, A., Hjalmers, I., Nevonen, L. (2001). Eating disorders, attachment and interpersonal difficulties: A comparison between 18- to 24-year-old patients and normal controls. 9,381-396. DOI:10.1002/erv.421

Carrasco, D., Gómez, E., \& Staforelli A. (2009). Obesidad y Adolescencia: Exploración de Aspectos Relacionales y Emocionales. Terapia psicológica, 27(1), 143-149.

Cassidy, J. (1994). Emotion regulation: Influences of attachment relationships. Monographs Of The Society For Research In Child Development, 59(2-3), 228283

Castillo, C. \& Romo, M. (2006). Las golosinas en la alimentación infantil. Revista chilena de pediatría, 77189 193. Recuperado en http://dx.doi.org/10.4067/ S0370-41062006000200011

Castillo-Durán C., Le Royo C., \& Osorio J. (2012) Obesidad síndrome metabólico en niños y adolescentes. Revista Médica Las Condes, 23(2) 160-164

Christakis, N. A. \& Fowler, H. J. (2007). The spread of obesity in large social network over 32 years. The New England Journal of Medicine, 357, 370-379.

Coromoto, M., Pérez, A., Herrera, H., Hernández, R. (2011). Hábitos Alimentarios, actividad física y su reacción con el estado nutricional - antropométrico de preescolares. Revista chilena de nutrición. ،38, 301-312.

Cruz, M., Tuñón, P., Villaseñor, F., Álvarez, G. \& Nigh, N. (2013). Sobrepeso y obesidad: una propuesta de abordaje desde la sociología. Revista Región y sociedad, 25 (57), 165-202.

de Lauzon, B., Romon, M., Deschamps, V., Lafay, L., Borys, J.M., Karlsson, J., et al. (2004). The Three-Factor Eating Questionnaire-R18 is able to distinguish among different eating patterns in a general population. The Journal of Nutrition, 134 (9), 2372-2380

Domínguez - Vásquez, Olivares \& Santos. (2009). Influencia familiar sobre la conducta alimentaria y su relación con la obesidad infantil. Archivos Latinoamericanos de Nutrición, 58, 249-255. Recuperado de http://repositorio.uchile.cl/bitstream/handle/2250/123925/Dominguez_p. pdf? sequence $=1 \&$ is Allowed $=y$

Encuesta Nacional de Consumo Alimentario (ENCA). (2014). Encuesta nacional de Consumo Alimentario. (s.n.) Recuperado de http://web.minsal.cl/sites/default/ files/ENCA-INFORME_FINAL.pdf.

Summa Psicológica UST (CC - BY - 3.0) ISSN: 0718-0446 / ISSNe: 0719-448x http://summapsicologica.c// 
Faber, A. (2015) Parental attachment insecurity predict schild and adult high-caloric food consumption. Journal of Health Psychology. 20, 511-524.

UNICEF. (2015). $4^{\circ}$ Estudio de Maltrato infantil en Chile: Análisis comparativo 1994-2000-2006-2012. Santiago de Chile.

Frankel, L. A., Hughes, S. O., O'Connor, T. M., Power, T. G., Fisher, J. O., \& Hazen, N. L. (2012). Parental influences on children's self-regulation of energy intake: Insights from developmental literature on emotion regulation. Journal of Obesity. Id 327259, Doi:10.1155/2012/327259

Guidano, V. (1987). Complexity Of The Self. Barcelona: Paidós.

Guidano, V. (1994). El Sí mismo en proceso. Barcelona: Paidós.

Guidano VF, Liotti G (1983). Cognitive Processes and Emotional Disorders. New York: Guilford

Guillén, R. (2014). Psicología de la Obesidad. Zaragoza: El Manual Moderno.

Henriquez, S., Basso, R. \& Alves, C. (2015). Relação entre Apego e Obesidade: Revisão Sistemática da Literatura., Psico, 46(1), 6-15.

Hesse, E. (1999). The adult attachment interview: Historical and current perspectives. En: J. Cassidy \& P. R. Shaver (Eds.), Handbook of attachment: theory, research and clinical applications, (pp. 395-443). New York: Guilford Press.

Karnik, S. \& Kanekar, A. (2012). Childhood Obesity: A Global Public Health Crisis. Revista Internacional de Medicina Preventiva, 3, 1-7. Recuperado de http://www. ncbi.nlm.nih.gov/pmc/articles/PMC3278864/

Kiesewetter, S., Köpsel, A., Mai, K., Stroux, A., Bobbert, T., Spranger, J., ... \& Kallenbach-Dermutz, B. (2012). Attachment style contributes to the outcome of a multimodal lifestyle intervention. BioPsychoSocial medicine, 6), 3, DOI: 10.1186/1751-0759-6-3

Manco, M., \& Dallapiccola, B. (2012). Genetics of pediatric obesity. Pediatrics, 130(1), 123-133.

Martínez, Olhaverry \& Cruzat. (2009). Aportes de la teoría del apego al abordaje clínico de los trastornos de alimentación. Revista argentina de clínica psicológica. Volumen (18) (pag. 2) Recuperado de http:// www.researchgate.net/publication/264421216

Mediano, (2015). Políticas Públicas en Alimentación y Nutrición: Nueva Ley de Etiquetado Vol. (3). Recuperado del sitio de internet de http://www.fch.cl/wp-content/uploads/2014/11/Fernanda_Mediano_Politicas_Publicas_en_Alimentacion_y_Nutricion_.pdf

Mikulincer, M. \& Shaver, PR. (2012). Adult Attachment Orientations and Relationship Processes. Journal of Family Theory \& Review, 4(4), 259-274. Recuperado en http://doi.org/10.1111/j.1756-2589.2012. 00142.x

Summa Psicológica UST (CC - BY - 3.0)

ISSN: 0718-0446 / ISSNe: 0719-448x

http://summapsicologica.cl/
Ministerio de Educación, JUNAEB, (2013). Informe Mapa Nutricional 2013. [Mensaje de página web]. Recuperado de: http://www.junaeb.cl/wp-content/ uploads/2013/03/Informe-Mapa-Nutricional-2013. pdf.henri

Ministerio de Salud. (2013). Estudio para la revisión y actualización de las guías alimentarias para la población chilena. Santiago.

Ministerio de Salud (MINSAL): Informe sobre la Encuesta nacional de salud, Ministerio de Salud, Santiago 2004.

Montalvini, P., Lucero, M., López, G. (2014). Estilos de apego y su relación con el patrón alimenticio de restricción-sobrealimentación en sujetos dietantes crónicos. Revista Chilena de Neuropsicología. 9(1-2), 8-11.

Morales P., Santos, J., Gonzales, A., Ho, J., Hodgson, M. (2012). Validación factorial de un cuestionario para medir la conducta de comer en ausencia de hambre y su asociación con obesidad infantil. Revista Chilena de Pediatría, 83 (5), 431-437.

Moreno, M. (2012). Definición y clasificación de la obesidad. Revista Médica Clínica Las Condes. 23 124-128. DOI: 10.1016/S0716-8640(12)70288-2

Oliva, A. (1995). Estado actual de la teoría del apego. Apuntes de Psicología, 45, 21-40. Recuperado de http://chitita.uta.cl/cursos/2012-1/0000636/recursos/r-9. pdf

Organización de las Naciones Unidas para la alimentación y la agricultura (2015). Panorama de la inseguridad Alimentaria en América Latina y el Caribe. Recuperado de http://www.fao.org/3/a-i4636s.pdf

Organización Mundial de la Salud, (2015). El sobrepeso y la obesidad se definen como una acumulación anormal o excesiva de grasa que puede ser perjudicial para la salud [Mensaje de página web]. Recuperado de http://www.who.int/mediacentre/factsheets/fs311/es/

Organizacion Panamericana de la Salud. (2000). La Obesidad en la Pobreza: Un nuevo reto para la salud pública. Washington, DC.

Osorio, J., Weisstaub, G. \& Castillo, C. (2002) Desarrollo de la conducta alimentaria en la infancia y sus alteraciones. Revista Chilena de Nutrición. Vol. 29, n.3. Recuperado de http://www. scielo.cl/scielo.php?script=sci_arttext\&pid $=$ S0717-75182002000300002

Pataky, Z., Robbioni-Harsch, E., Golay, A. (2010). Obesity: A ComplexGrowingChallenge. Exp Clin Endocrinol Diabetes. 2010; 118: 427 - 43Peña, M., \& Bacallao, J. (2001). La obesidad y sus tendencias en la Región. Revista Panamericana de Salud Publica, 10(2), 75-78. 
Pinedo Palacios, J R, Santelices Álvarez, M P. (2006). Apego adulto: Los Modelos Operantes Internos y la Teoría de la Mente. Terapia Psicológica, 24,201-209. Recuperado de http://www.redalyc.org/articulo. oa?id $=78524210$

Procaccia, R., Veronese, G. \& Castiglioni, M. (2014). The Impact of Attachment Style on the Family Drawings of School-Aged Children. The Open Psychology Journal. 7, 9-17.

Schaffer, H. R. \& Emerson, P. E. (1964). Patterns of response to physical contact in early human development. Journal of Child Psychology and Psychiatry, 5(1), $1-13$.

Valladares, M., Obregón, A., Weisstaub, G., Burrows, R., Patiño, A., Ho-Urriola, J. \& Santos, J. (2015). Asociación entre la conducta alimentaria y polimorfismos genéticos de la leptina y su receptor en niños obesos chilenos. Nutrición Hospitalaria. 31,1044-1051.

Van der Merve, M-T. (2007). Psychological correlates of obesity in women. International Journal of Obesity, 31(Suppl. 2), S14-S18.

Vázquez, V. (2004). ¿Cuáles son los problemas psicológicos, sociales y familiares que deben ser considerados en el diagnóstico y tratamiento del paciente obeso? Revista de Endocrinología y Nutrición, 12(3), 136-142.

Zagmutt, A. (2010). Vínculos Afectivos, Mentes Conectadas. Santiago: Uqbar. 